\title{
High-level mupirocin resistance in methicillin-resistant staphylococci isolated from dogs and cats
}

\author{
Magdalena Kizerwetter-Świda* (i), Dorota Chrobak-Chmiel and Magdalena Rzewuska
}

\begin{abstract}
Background: Mupirocin is one of the few antimicrobials active against methicillin-resistant Staphylococcus aureus (MRSA), and is frequently used for the eradication of MRSA nasal colonisation in humans. Initially, mupirocin resistance was recognised in human $S$. aureus, including MRSA isolates, then also among coagulase-negative staphylococci (CoNS). Nowadays, mupirocin resistance is occasionally observed in canine staphylococci, along with Staphylococcus pseudintermedius (MRSP) strains, as well as CoNS, which usually show methicillin resistance. In the current study, high-level mupirocin resistance in methicillin-resistant staphylococci isolated from diseased dogs and cats was investigated.
\end{abstract}

Results: Among 140 methicillin-resistant staphylococci isolates from dogs and cats, three showed high-level mupirocin resistance in a screening test using the agar disk diffusion method. One was recognised as methicillinresistant S. aureus, one as methicillin-resistant S. pseudintermedius, and one as methicillin-resistant Staphylococcus haemolyticus. S. pseudintermedius and S. aureus were isolated from dogs, S. haemolyticus was obtained from a cat. All isolates showed high-level mupirocin resistance, confirmed by minimum inhibitory concentration (MIC) values of above $1024 \mathrm{\mu g} / \mathrm{ml}$ and the presence of the plasmid-located gene iles2. This is the first report on the detection of high-level mupirocin resistance (HLMR) in S. haemolyticus of feline origin.

Conclusions: This study revealed the occurrence of HLMR in three Staphylococcus isolates obtained from companion animals in Poland. The results of this study indicate that the monitoring of mupirocin resistance in staphylococci of animal origin, especially in methicillin-resistant isolates, is strongly recommended.

Keywords: Staphylococcus aureus, Staphylococcus pseudintermedius, Staphylococcus haemolyticus, ileS2

\section{Background}

Staphylococci are extremely versatile and constantly evolving microorganisms. One of the major concerns for public health is the spread of methicillin resistance within the Staphylococcus genus. Worryingly, methicillin-resistant strains typically also show multidrug resistance, in addition to resistance to virtually all $\beta$-lactam antibiotics [1-4]. Mupirocin is a topical antimicrobial used to treat superficial bacterial skin infections and to control the spread of methicillin-resistant Staphylococcus aureus (MRSA) in humans. Mupirocin resistance was at first recognised in $S$. aureus strains, and it is well characterised within this

\footnotetext{
*Correspondence: magdalena_kizerwetter_swida@sggw.pl

Division of Microbiology, Department of Preclinical Sciences, Faculty of

Veterinary Medicine, Warsaw University of Life Sciences, Ciszewskiego, 802-786 Warsaw, Poland
}

species. Two mupirocin-resistant phenotypes have been described: low-level and high-level resistance [5]. Low-level mupirocin resistance (LLMR) is attributed to a point mutation or mutations in the chromosomal ileS gene encoding isoleucyl-tRNA synthetase. Strains with such mutations are characterised by low mupirocin minimum inhibitory concentration (MIC) values falling within the range from $\geq 8 \mu \mathrm{g} / \mathrm{ml}$ to $256 \mu \mathrm{g} / \mathrm{ml}$ [6]. The mechanism of high-level mupirocin resistance (HLMR) is associated with a conjugative plasmid carrying the ileS2 (mupA) gene encoding an additional isoleucyl-tRNA synthetase with reduced affinity for mupirocin. The MIC of mupirocin in the case of HLMR is $\geq 512 \mu \mathrm{g} / \mathrm{ml}$ [5]. Initially, mupirocin was used in human medicine, especially for the elimination of MRSA nasal colonisation and soon the increased prevalence of resistance was observed. After MRSA hospital outbreaks and frequent 
decolonisation in patients and the medical staff using mupirocin, resistance was even observed in up to $63 \%$ of locally isolated methicillin-resistant strains [7]. Moreover, mupirocin resistance was also found in coagulase-negative staphylococci (CoNS) [8].

In veterinary medicine, the increasing prevalence of infections caused by methicillin-resistant staphylococci is becoming a worrying issue. The most widespread methicillin-resistant species in dogs is Staphylococcus pseudintermedius (MRSP), but MRSA, as well as methicillin-resistant CoNS, are also isolated from companion animals $[1-3,9]$. Generally, these strains show multidrug-resistance, significantly limiting the treatment options, and leading to the more frequent use of alternative antimicrobials, like mupirocin [4, 10-13]. Although mupirocin is only approved for use in animals in some countries, such as the United States [10], off-label use is a possibility for veterinarians. In Poland, mupirocin is not registered for use in animals. Nowadays, mupirocin resistance occasionally occurs in staphylococci isolated from companion animals [14-19]. However, it may be assumed, that these data are understated because susceptibility to mupirocin is not routinely determined for animal isolates. However, there is a lack of data regarding high-level mupirocin resistance in staphylococci of animal origin in Poland. The aim of this study was to investigate the prevalence and the mechanism of highlevel mupirocin resistance among methicillin-resistant staphylococci isolated from diseased dogs and cats.

\section{Results}

\section{Prevalence of HLMR among methicillin-resistant staphylococci}

Of the 140 methicillin-resistant staphylococci isolated between 2007 and 2017, three (2.1\%) isolates showed HLMR in a screening test using the agar disk diffusion method (Confidence interval, CI 95\%: 0.7-6.1\%) (Additional file 1: Figure S1). Two were obtained from dogs and one from a cat, in 2007, 2013, and 2016 respectively.

\section{Identification of high-level mupirocin-resistant staphylococcal isolates}

Based on the results of standard bacteriological tests, all isolates were recognised as staphylococci, two were coagulase-positive, and one coagulase-negative. Using a nuc-specific PCR, one of the two coagulase-positive isolates was classified as $S$. aureus and the other as $S$. pseudintermedius (Additional file 1: Figure S2). The single coagulase-negative isolate was identified as Staphylococcus haemolyticus with APIStaph with a reliability of $82.2 \%$ and confirmed by sequence analysis of the $16 \mathrm{~S}$ rRNA gene. According to the BLAST analysis, the sequence of the 16S rRNA gene displayed 100\% concordance with a type strain of S. haemolyticus ATCC 29970
(GenBank: D83367.1), confirming the identification of our isolate as S. haemolyticus. The characteristics of the isolates are shown in Table 1.

\section{Antimicrobial susceptibility}

All three isolates tested showed resistance to $\beta$-lactam antibiotics evaluated by the agar disk diffusion method: penicillin, amoxicillin, amoxicillin with clavulanic acid, ceftiofur, and oxacillin or cefoxitin according to the species of staphylococci tested. The $m e c A$ gene was detected in all isolates, confirming their methicillin resistance (Additional file 1: Figure S3). The results of the tests for antimicrobial susceptibility testing by agar disk diffusion method for non- $\beta$-lactam antibiotics using the agar disk diffusion method are presented in Table 2. All three isolates showed the multidrug- resistance phenotype with resistance to $\beta$-lactam antibiotics, fluoroquinolones, macrolides, lincosamides, and were highlevel mupirocin resistant. Detailed resistance profiles for each strain are given in Table 2.

\section{Mupirocin resistance}

High-level mupirocin resistance was confirmed in all three staphylococcal isolates. The MIC values of mupirocin for the isolates were above $1024 \mu \mathrm{g} / \mathrm{ml}$ (Table 2). A specific ileS2 gene fragment of $458 \mathrm{bp}$ was detected in PCR for all isolates tested (Additional file 1: Figure S4). Comparison of the ileS2 sequences of the three examined isolates revealed $100 \%$ identity with the previously published sequence of plasmidic ileS2 gene from the mupirocin-resistant S. pseudintermedius strain HR547/ 11 (GeneBank: JX186508).

\section{Discussion}

The three Staphylococcus isolates obtained from companion animals during routine bacteriological examination showed multidrug resistance, as well as methicillin and mupirocin resistance. Furthermore, the MIC value of mupirocin $\geq 1024 \mu \mathrm{g} / \mathrm{ml}$ and the presence of the ileS2 gene confirmed HLMR in all isolates tested. The antimicrobial treatment history of the animals from which the isolates were obtained was not available; therefore, the impact of previous antibiotic use on the selection of the mupirocin-resistant resistant staphylococci cannot be assessed. Our results confirmed the occasional occurrence of high-level mupirocin resistance in staphylococci of animal origin reported previously by others. Recently, one MRSP isolate out of $110 \mathrm{~S}$. pseudintermedius canine isolates tested in Korea showed HLMR [16]. Similarly, resistance to mupirocin was found in one out of $100 \mathrm{~S}$. pseudintermedius isolates from healthy dogs in Australia, and this strain was also multidrug resistant [15]. In the USA, among $581 \mathrm{~S}$. pseudintermedius, HLMR determined by the plasmidic ileS 2 gene was found in one 
Table 1 The characteristics of Staphylococcus isolates with HLMR used in the study

\begin{tabular}{lllll}
\hline Isolate ID & Origin & Type of clinical sample & mecA presence & Identification \\
\hline $583 / 07$ & Dog & Bursa exudate & + & S. aureus/MRSA \\
$813 / 13$ & Dog & Wound swab & + & S. pseudintermedius/MRSP \\
$840 / 16$ & Cat & Abscess & + & S. haemolyticus/MRSH \\
\hline
\end{tabular}

methicillin-susceptible Staphylococcus pseudintermedius (MSSP) isolate [14]. Matanovic et al. described HLMR in one out of $102 \mathrm{~S}$. pseudintermedius strains isolated from dogs in Croatia [17]. This strain was classified as MSSP, the ileS2 gene was located on a conjugative plasmid, which additionally contained the aminoglycoside resistance $a a c A-a p h D$ gene. In another study conducted in the USA, two mupirocin-resistant strains, one MRSP, and one methicillin-resistant Staphylococcus sciuri, were found among staphylococci isolated from dogs with superficial pyoderma. Studies conducted in England showed one canine MRSA in 204 examined S. aureus with a MIC of mupirocin $16 \mu \mathrm{g} / \mathrm{ml}$, consistent with values assigned as low-level mupirocin resistance. In contrast to these results, in Canada all S. pseudintermedius isolates obtained from dogs with skin and soft tissue infections $(n=$ 50) demonstrated susceptibility to mupirocin.

Antimicrobial resistance genes are easily transferred between staphylococci even among different species of the genus, which is particularly evident in the dissemination of the mecA gene [4]. Likewise, plasmid-mediated HLMR can disseminate horizontally and clonally. In vitro and in vivo transfer of the ileS2 gene between $S$. aureus and Staphylococcus epidermidis was described [20]. Moreover, plasmids conferring the ileS2 gene may also contain genes determining gentamicin, tetracycline or macrolide resistance. Exposure to any of these antimicrobials would coselect for the resistant strains. The three high-level mupirocin-resistant and methicillinresistant isolates described in this study showed different resistance patterns to other antimicrobials; all were resistant to fluoroquinolones, macrolides, and lincosamides.

The emergence of mupirocin resistance among MRSA and other staphylococci in humans suggests that it may be potentially transferred to staphylococci of animal origin. The close contacts of owners with their dogs and cats favour the spread of resistant bacteria, including mupirocin-resistant ones. The transmission of mupirocin resistance genes from human S. aureus isolates to canine S. pseudintermedius is highly probable, although it may occur in both directions. However, selective pressure is the main factor contributing to the rise and increase in antimicrobial resistance. Recently, MRSP and other methicillin-resistant staphylococci have emerged as important pathogens in small animal veterinary medicine $[1-3]$. These bacteria typically show multidrug resistance, which significantly limits effective treatment options [11, 12, 21]. Topical mupirocin use in companion animals provides some therapeutic options for infections caused by methicillin-resistant strains, thus mupirocin use in veterinary practice may also increase in the coming years [13]. However, the use of this antimicrobial in veterinary medicine raises some concerns related to the idea that mupirocin should be reserved only for human medicine. Counteracting the increase in antimicrobial resistance including mupirocin can only be achieved through complex and coordinated actions, such as the One Health approach.

\section{Conclusions}

In conclusion, this is the first report describing HLMR in multidrug-resistant and methicillin-resistant staphylococci isolated from companion animals in Poland. To our knowledge, this is also the first description of HLMR in S. haemolyticus of feline origin. Mupirocin resistance was previously found in $S$. haemolyticus strain isolates from humans and dogs $[15,22]$. Imprudent antimicrobial use could cause HLMR strains to become more prevalent among animals in the future. The incidence of resistant bacteria is an important public health threat,

Table 2 The results of antimicrobial susceptibility testing to non- $\beta$-lactam antibiotics for studied staphylococcal isolates

\begin{tabular}{|c|c|c|c|}
\hline \multirow[t]{2}{*}{ Isolate ID/Species } & \multicolumn{2}{|l|}{ Disk diffusion test } & \multirow{2}{*}{$\begin{array}{l}\mathrm{MIC}(\mu \mathrm{g} / \mathrm{ml}) \\
\text { of mupirocin }\end{array}$} \\
\hline & Resistant & Susceptible & \\
\hline $\begin{array}{l}583 / 07 \\
\text { S. aureus }\end{array}$ & ENR, MAR, TET, ERY, L, RIF, MUP & GEN, SXT, CHL & $>1024$ \\
\hline $\begin{array}{l}813 / 13 \\
\text { S. pseudintermedius }\end{array}$ & SXT, ENR, MAR, TET, ERY, GEN, L, CHL, MUP & RIF & $>1024$ \\
\hline $\begin{array}{l}840 / 16 \\
\text { S. haemolyticus }\end{array}$ & SXT, ENR, MAR, ERY, GEN, L, MUP & TET, RIF, CHL & $>1024$ \\
\hline
\end{tabular}

SXT-sulfamethoxazole/trimethoprim, TET-tetracycline, GEN-gentamicin, ENR-enrofloxacin, MAR-marbofloxacin, ERY-erythromycin, CHL-chloramphenicol, Llincomycin, RIF-rifampicin, MUP-mupirocin 
thus surveillance of resistance, including mupirocin resistance among animal staphylococci, is strongly recommended. Moreover, the presence of HLMR among staphylococci obtained from the companion animals is of public health concern and emphasises the need for the introduction of antimicrobial stewardship programmes in veterinary settings.

\section{Methods}

\section{Bacterial isolates}

A collection of 140 methicillin-resistant staphylococci was investigated for the presence of high-level mupirocin resistance. All the isolates used in this study were obtained from clinical specimens of animal origin submitted to the Microbiological Diagnostic Laboratory, Faculty of Veterinary Medicine, Warsaw University of Life Sciences-SGGW in Poland during routine bacteriological examinations between 2007 and 2017. The types of clinical samples from which staphylococci were isolated are given in Table 1. The collection comprised of 126 canine and 14 feline isolates. The identification of staphylococci was based on standard bacteriological methods: colony morphology, Gram staining, catalase testing, coagulase production, and the slide agglutination test. Bacterial DNA was isolated using a DNA Genomic Mini kit (A\&A Biotechnology) and lysostaphin $(100 \mathrm{mg} /$ $\mathrm{ml}$ ) according to the manufacturer's instructions. The amount and quality of DNA were determined using The Thermo Scientific NanoDrop ${ }^{\text {tw1 }} 1000$ Spectrophotometer. The identification of coagulase-positive staphylococci was confirmed by nuc gene PCR analysis [23]. The identification of the coagulase-negative isolate was based on the biochemical properties determined with APIStaph and confirmed by PCR amplification and sequence analysis of the 16S rRNA gene using universal primers [24]. The amplicon was sequenced using a $3730 \mathrm{xl}$ DNA Analyzer. Sequencing files were analysed using the Chromas Lite version 2.33 program. Nucleotide BLAST analysis was carried out on the National Center for Biotechnology Information (NCBI) website (http://blast. ncbi.nlm.nih.gov).

\section{Antimicrobial susceptibility testing}

Methicillin resistance was ascertained using the agar disk diffusion method with oxacillin (OXA-1 $\mu \mathrm{g}$ ) or cefoxitin (FOX-30 $\mu \mathrm{g}$ ) depending on the staphylococcal species [25]. Methicillin resistance was confirmed by $m e c A$ gene amplification by PCR as described by Strommenger et al. [26]. High-level mupirocin resistance was initially detected according to the method recommended by CLSI using $200 \mu \mathrm{g}$ mupirocin disks [27]. Isolates showing no zone around the disk were regarded as high-level mupirocin-resistant and subjected to further testing. The 95\% Confidence Interval was calculated using the
Wilson score method [28]. The high-level mupirocinresistant isolates were subjected to routine antimicrobial susceptibility testing using the agar disk diffusion method for a panel of the following antimicrobials: penicillin, amoxicillin, amoxicillin with clavulanic acid, ceftiofur, sulfamethoxazole/trimethoprim, tetracycline, gentamicin, enrofloxacin, marbofloxacin, and erythromycin. Extended susceptibility testing was performed for: mupirocin, chloramphenicol, lincomycin, and rifampicin. The CLSI veterinary guidelines [25] were used for interpretation of the agar disk diffusion testing results.

\section{Minimal inhibitory concentration of mupirocin}

After the initial detection of HLMR using $200 \mu \mathrm{g}$ mupirocin disk, the minimal inhibitory concentration of mupirocin was determined using the broth microdilution technique for $0.064-1024 \mu \mathrm{g} / \mathrm{ml}$ of mupirocin (Sigma) according to the CLSI guidelines [27].

\section{Detection of mupirocin resistance by PCR}

The ileS2 gene determining high-level mupirocin resistance was detected by PCR method described by Anthony et al. [29] using the primers ileS2F and ileS2R to amplify a 458 bp fragment of the ileS2 gene.

\section{Sequence analysis}

To confirm the PCR results, the obtained amplicons were sequenced using the primers forward and reverse. The sequences were compared with the ileS2 sequences available in the Genbank using the nucleotide BLAST program (http://blast.ncbi.nlm.nih.gov).

\section{Additional file}

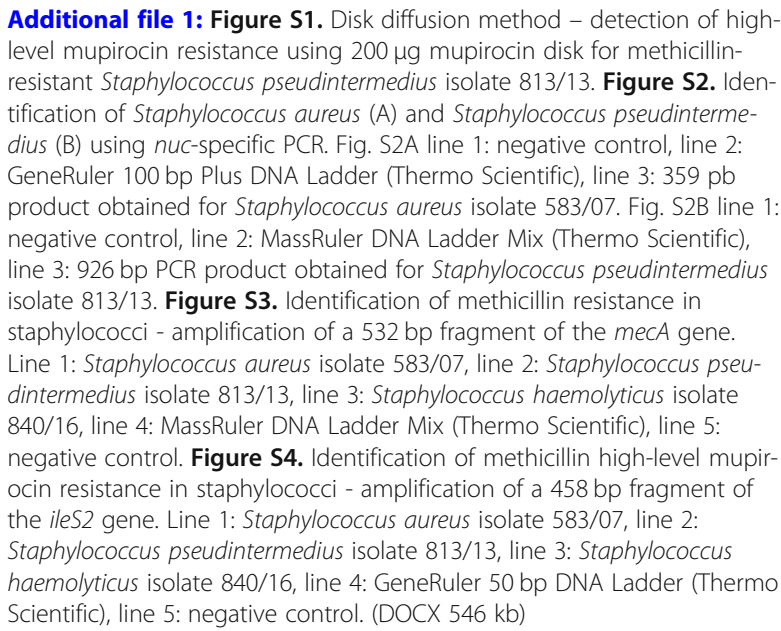

Additional file 1: Figure S1. Disk diffusion method - detection of highlevel mupirocin resistance using $200 \mu \mathrm{g}$ mupirocin disk for methicillinresistant Staphylococcus pseudintermedius isolate 813/13. Figure S2. Identification of Staphylococcus aureus (A) and Staphylococcus pseudintermedius (B) using nuc-specific PCR. Fig. S2A line 1: negative control, line 2: GeneRuler 100 bp Plus DNA Ladder (Thermo Scientific), line 3: 359 pb product obtained for Staphylococcus aureus isolate 583/07. Fig. S2B line 1: negative control, line 2: MassRuler DNA Ladder Mix (Thermo Scientific), line 3: 926 bp PCR product obtained for Staphylococcus pseudintermedius isolate $813 / 13$. Figure S3. Identification of methicillin resistance in staphylococci - amplification of a 532 bp fragment of the mecA gene. Line 1: Staphylococcus aureus isolate 583/07, line 2: Staphylococcus pseudintermedius isolate 813/13, line 3: Staphylococcus haemolyticus isolate 840/16, line 4: MassRuler DNA Ladder Mix (Thermo Scientific), line 5: negative control. Figure S4. Identification of methicillin high-level mupirocin resistance in staphylococci - amplification of a $458 \mathrm{bp}$ fragment of the iles2 gene. Line 1: Staphylococcus aureus isolate 583/07, line 2: Staphylococcus pseudintermedius isolate 813/13, line 3: Staphylococcus haemolyticus isolate 840/16, line 4: GeneRuler 50 bp DNA Ladder (Thermo Scientific), line 5: negative control. (DOCX 546 kb)

\section{Abbreviations}

AMC: Amoxicillin with clavulanic acid; AMX: Amoxicillin; CHL: Chloramphenicol; CLSI: Clinical and Laboratory Standards Institute; CoNS: Coagulase-negative staphylococci; ENR: Enrofloxacin; 
ERY: Erythromycin; FOX: Cefoxitin; GEN: Gentamicin; HLMR: High-level mupirocin resistance; L: Lincomycin; MAR: Marbofloxacin; MIC: Minimum inhibitory concentration; MRSA: Methicillin-resistant Staphylococcus aureus MRSH: Methicillin-resistant Staphylococcus haemolyticus; MRSP: Methicillinresistant Staphylococcus pseudintermedius; MSSP: Methicillin-susceptible Staphylococcus pseudintermedius; MUP: Mupirocin; OXA: Oxacillin; PEN: Penicillin; RIF: Rifampicin; SXT: Sulfamethoxazole/trimethoprim; TET: Tetracycline; XNL: Ceftiofur

\section{Acknowledgments}

The authors thank Barbara Chojnacka and Alicja Grzechnik for excellent technical assistance.

\section{Authors' contributions}

MKŚ was involved in study design and coordination, data management and performed all laboratory analyses and interpretation as well as preparation of the manuscript draft. DCC participated in designing of the study, in the laboratory, assisted in the editing of the manuscript. MR participated in designing of the study, in the laboratory, assisted in the editing of the manuscript. All authors commented on the manuscript and approved the final version.

\section{Funding}

Not applicable.

\section{Availability of data and materials}

All data obtained and analyzed in this study are included in this manuscript. The additional figures can be found in Additional file 1.

\section{Ethics approval and consent to participate}

Not applicable.

\section{Consent for publication}

Not applicable.

\section{Competing interests}

The authors declare that they have no competing interests.

Received: 5 February 2019 Accepted: 23 June 2019

Published online: 10 July 2019

\section{References}

1. Perreten V, Kadlec K, Schwarz S, Grönlund Andersson U, Finn M, Greko C, Moodley A, Kania SA, Frank LA, Bemis DA, Franco A, lurescia M, Battisti A, Duim B, Wagenaar JA, van Duijkeren E, Weese JS, Fitzgerald JR, Rossano A, Guardabassi L. Clonal spread of methicillin-resistant Staphylococcus pseudintermedius in Europe and North America: an international multicentre study. J Antimicrob Chemother. 2010;65:1145-54.

2. Ruscher C, Lübke-Becker A, Semmler T, Wleklinski CG, Paasch A, Soba A Stamm I, Kopp P, Wieler LH, Walther B. Widespread rapid emergence of a distinct methicillin- and multidrug-resistant Staphylococcus pseudintermedius (MRSP) genetic lineage in Europe. Vet Microbiol. 2010;144:340-6.

3. Moodley A, Damborg P, Nielsen SS. Antimicrobial resistance in methicillin susceptible and methicillin resistant Staphylococcus pseudintermedius of canine origin: literature review from 1980 to 2013 Vet Microbiol. 2013;171: 337-341.

4. McCarthy AJ, Harrison EM, Stanczak-Mrozek K, Leggett B, Waller A, Holmes MA, Lloyd DH, Lindsay JA, Loeffler A. Genomic insights into the rapid emergence and evolution of MDR in Staphylococcus pseudintermedius. J Antimicrob Chemother. 2015:70:997-1007.

5. Patel JB, Gorwitz RJ, Jernigan JA. Mupirocin resistance. Clin Infect Dis. 2009; 49:935-41.

6. Lee AS, Gizard Y, Empel J, Bonetti EJ, Harbarth S, François P. Mupirocininduced mutations in iles in various genetic backgrounds of methicillinresistant Staphylococcus aureus. J Clin Microbiol. 2014:52:3749-54.

7. Netto dos Santos KR, de Souza Fonseca L, Gontijo Filho PP. Emergence of high-level mupirocin resistance in methicillin-resistant Staphylococcus aureus isolated from Brazilian university hospitals. Infect Control Hosp Epidemiol. 1996;17:813-6

8. Rossi CC, Ferreira NC, Coelho ML, Schuenck RP, Bastos Mdo C. GiambiagideMarval M. 2016. Transfer of mupirocin resistance from Staphylococcus haemolyticus clinical strains to Staphylococcus aureus through conjugative and mobilizable plasmids. FEMS Microbiol Lett. 2016. https://doi.org/10. 1093/femsle/fnw121.

9. Couto N, Monchique C, Belas A, Marques C, Gama LT, Pomba C. Trends and molecular mechanisms of antimicrobial resistance in clinical staphylococci isolated from companion animals over a 16 year period. J Antimicrob Chemother. 2016;71:1479-87.

10. Werner A, Russel D. Mupirocin, fusidic acid and bacitracin: activity, action and clinical uses of three topical antibiotics. Vet Dermatol. 1999;10:225-40

11. Bond R, Loeffler A. What's happened to Staphylococcus intermedius? Taxonomic revision and emergence of multi-drug resistance. J Small Anim Pract. 2012:53:147-54

12. Frank LA, Loeffler A. Methicillin-resistant Staphylococcus pseudintermedius: clinical challenge and treatment options. Vet Dermatol. 2012;23:283-91.

13. Valentine BK, Dew W, Yu A, Weese JS. In vitro evaluation of topical biocide and antimicrobial susceptibility of Staphylococcus pseudintermedius from dogs. Vet Dermatol. 2012. https://doi.org/10.1111/j.1365-3164.2012.01095.x.

14. Godbeer SM, Gold RM, Lawhon SD. Prevalence of mupirocin resistance in Staphylococcus pseudintermedius. J Clin Microbiol. 2014;52:1250-2.

15. Bean DC, Wigmore M. Carriage rate and antibiotic susceptibility of coagulase-positive staphylococci isolated from healthy dogs in Victoria, Australia. Aust Vet J. 2016;94:456-60.

16. Park JH, Kang JH, Hyun JE, Hwang CY. Low prevalence of mupirocin resistance in Staphylococcus pseudintermedius isolates from canine pyoderma in Korea. Vet Dermatol. 2018. https://doi.org/10.1111/vde.12518.

17. Matanovic K, Pérez-Roth E, Pintarić S, Šeol Martinec B. Molecular characterization of high-level mupirocin resistance in Staphylococcus pseudintermedius. J Clin Microbiol. 2013;51:1005-7.

18. Fulham KS, Lemarie SL, Hosgood G, Dick HL. In vitro susceptibility testing of meticillin-resistant and meticillin-susceptible staphylococci to mupirocin and novobiocin. Vet Dermatol. 2011:22:88-94.

19. Loeffler A, Baines SJ, Toleman MS, Felmingham D, Milsom SK, Edwards EA, Lloyd DH. In vitro activity of fusidic acid and mupirocin against coagulasepositive staphylococci from pets. J Antimicrob Chemother. 2008:62:1301-4

20. Hurdle JG, O'Neill AJ, Mody L, Chopra I, Bradley SF. In vivo transfer of highlevel mupirocin resistance from Staphylococcus epidermidis to methicillinresistant Staphylococcus aureus associated with failure of mupirocin prophylaxis. J Antimicrob Chemother. 2005:56:1166-8.

21. Kizerwetter-Świda M, Chrobak-Chmiel D, Rzewuska M, Binek M. Resistance of canine methicillin-resistant Staphylococcus pseudintermedius strains to pradofloxacin. J Vet Diagn Investig. 2016;28:514-8.

22. Bean DC, Wigmore SM, Wareham DW. Draft genome sequence of a canine isolate of methicillin-resistant Staphylococcus haemolyticus. Genome Announc. 2017. https://doi.org/10.1128/genomeA.00146-17.

23. Sasaki T, Tsubakishita S, Tanaka Y, Sakusabe A, Ohtsuka M, Hirotaki S, Kawakami T, Fukata T, Hiramatsu K. Multiplex-PCR method for species identification of coagulase-positive staphylococci. J Clin Microbiol. 2010;48: $765-9$.

24. Devereux R, Wilkinson SS. Amplification of ribosomal RNA sequences. In: Kowalchuk GA, de Bruijn F, Head IM, Van der Zijpp AJ, van Elsas JD, editors. Molecular microbial ecology manual. New York: Springer; 2004. p. 509-22.

25. Clinical and Laboratory Standards Institute (CLSI). Performance standards for antimicrobial disk and dilution susceptibility tests for bacteria isolated from animals: $4^{\text {th }}$ edition supplement VET08. Wayne: CLSI; 2018.

26. Strommenger B, Kettlitz C, Werner G, Witte W. Multiplex PCR assay for simultaneous detection of nine clinically relevant antibiotic resistance genes in Staphylococcus aureus. J Clin Microbiol. 2003;41:4089-94.

27. Clinical and Laboratory Standards Institute (CLSI). Performance standards for antimicrobial susceptibility testing: $28^{\text {th }}$ edition M100. Wayne: CLSI; 2018.

28. Altman D, Machin D, Bryant T, Gardner M. Statistics with confidence: confidence intervals and statistical guidelines, 2nd edn: BMJ books; 2000.

29. Anthony RM, Connor AM, Power EG, French GL. Use of the polymerase chain reaction for rapid detection of high-level mupirocin resistance in staphylococci. Eur J Clin Microbiol Infect Dis. 1999:18:30-4.

\section{Publisher's Note}

Springer Nature remains neutral with regard to jurisdictional claims in published maps and institutional affiliations. 